\title{
Low-loss multilayer compatible a-Si:H optical thin films for photonic applications
}

\author{
T. Lipka \\ timo.lipka@tu-harburg.de
}

O. Horn

\section{J. Amthor}

\section{J. Müller}

This paper reports about hydrogenated amorphous silicon which can be employed as low-loss optical material for small footprint and cost-effective photonic integrated circuits. Basic waveguides, photonic wire based couplers, Mach-Zehnder interferometers, ring resonators and Mach-Zehnder assisted ring resonators were designed, fabricated, and optically characterised. The propagation loss of rib and photonic wire waveguides were determined to be $2 \mathrm{~dB} / \mathrm{cm}$ and $5.3 \mathrm{~dB} / \mathrm{cm}$, respectively. The $90^{\circ}$ bending losses of $5 \mu \mathrm{m}$ curved photonic wires were determined to be $0.025 \mathrm{~dB} / 90^{\circ}$. Three-dimensional tapers, which were fabricated without additional etching steps and were deposited on top of the fabricated photonic wires showed a net coupling loss of $4 \mathrm{~dB} /$ port. Multimode $3 \mathrm{~dB}$-splitters were systematically investigated resulting in $49-51 \%$ splitting ratios. Mach-Zehnder interferometers that were realised with these splitters showed interference fringe depths of up to $25 \mathrm{~dB}$ for both polarisations. Compact ring resonators with $10 \mu \mathrm{m}$ radius implemented as notch filters and in Mach-Zehnder coupled configurations provided extinction ratios of $\geq 20 \mathrm{~dB}$ and Q-factors up to 7500 .

[DOI: http://dx.doi.org/10.2971/jeos.2012.12033]

Keywords: dielectric thin films, amorphous silicon, integrated optics, photonics, resonators

\section{INTRODUCTION}

Amorphous silicon dielectric thin films are widely used in industrial and consumer electronic applications and are already present in everyday life. However, thus far hydrogenated amorphous silicon (a-Si:H) is still rarely exploited as photonic material in the field of integrated optics, where it could be potentially used as a transparent material in the near infrared region for telecommunication and computing applications, or for chemical and biological diagnostics and sensing.

Presently, crystalline silicon (SOI) is the preferred material, although a-Si:H shares the same advantages as photonic integration platform, which is primarily due to two reasons. These are the compatibility to mature CMOS-fabrication technology, which reduces process development time and costs, and the high refractive index of the Si-materials, which permits a high integration density and hence photonic systems with low footprint, low power consumption, and functional devices covering a large free spectral range.

Among the Si-based high index materials SOI shows the highest optical quality in terms of material absorption [1], but exhibits limited multilayer capabilities. This restricts the flexibility in the design and the fabrication of three-dimensional applications and therefore the number of vertical interacting optical layers per chip. These aspects drive the increasing interest in the development of deposited optical thin film ma- terials like hydrogenated amorphous and poly silicon ( $\mathrm{p}-\mathrm{Si}$ ). However, p-Si waveguides still have to be treated with thermal annealing $\left(600-1100^{\circ} \mathrm{C}\right)$ in order to achieve low-loss material $[2,3]$. Compared to that, a-Si:H can be fabricated with low temperatures $\left(200-400^{\circ} \mathrm{C}\right)$ using plasma-enhanced chemical vapour deposition (PECVD) [4, 5]. The released thermal budget of this process directly meets backend fabrication requirements, which facilitates the multilayer stacking of photonic layers on top of integrated microchips in the last step of the CMOS process line. This will allow combining the benefits of integrated electronic circuits with the unique properties of high-speed optical data transmission by interconnecting both disciplines.

The rising interest is also reflected in the increasing amount of recent publications using a-Si:H material as photonic guiding layer. There are reports about low-loss photonic wires [6], about functional photonic components as ring resonators and interferometers $[7,8]$, and about active tuneable devices either electro- or all-optically $[9,10]$.

In this paper, we report about our recent research results in this area. Numerical calculations and simulations were performed in order to design functional photonic components like 3D-tapers, polarisation insensitive $3 \mathrm{~dB}$ power splitters, 
Mach-Zehnder interferometers, and ring resonators in notch and Mach-Zehnder coupled configuration.

A detailed investigation and optical measurements of the fabricated devices are presented. The multilayer capability of the PECV-deposition process is proven by fabricating 3D-etchless tapers. The high optical quality of the a-Si:H thin films is demonstrated with low-loss photonic waveguides and passive photonic components like Mach-Zehnder interferometers and ring resonators that are systematically analysed.

\section{PHOTONIC WAVEGUIDE AND SYSTEM DESGN}

In general, the behaviour of any wavelength-selective optical device (e.g. Mach-Zehnder interferometer, ring resonator) relies on the effective mode index $\left(n_{e f f}\right)$, which is defined by the materials refractive indices and the waveguide dimensions and shape. In high index contrast systems with $\Delta n \approx 2$ between silicon core and oxide cladding layers, $n_{e f f}$ is mostly dominated by the waveguide dimensions. The higher the index contrast between the waveguide core and cladding, the tighter the light confinement and hence the possible achievable integration density. However, a high refractive index contrast implies strong demands in fabrication, especially with regard to surface and sidewall roughness, and the waveguide dimension accuracy $[11,12]$.

In this work, a full-vectorial finite element mode solver (COMSOL Multiphysics) was used for the optical simulations and was employed for the waveguide and photonic component designs.

\subsection{Waveguide Design}

For most optical applications, the single-mode operation is preferred, which limits the maximal size of the waveguides. In order to meet this criterion for sub- $\mu \mathrm{m}$ channel waveguides the size of our photonic wires was restricted to $\mathrm{w} \times \mathrm{h} \leq 500 \times 220 \mathrm{~nm}$, thus only the fundamental transversal electric and transversal magnetic modes (qTE,qTM) are guided [13]. For a photonic wire geometry of $480 \times 220 \mathrm{~nm}$ the effective index is calculated to be $n_{e f f} \approx 2.41$ for qTE and $n_{\text {eff }} \approx 1.76$ for qTM mode at $1550 \mathrm{~nm}$ when taking an oxide upper cladding for the simulations. Compared to photonic wires, properly designed rib waveguides can be still monomode with dimensions of several $\mu \mathrm{m}$, which makes the measurement of propagation loss and an estimation of bulk material loss much easier and more reliable than with highly sensitive photonic wires $[14,15]$.

For an accurate design of photonic wire based spectral devices the group refractive index is considered as it takes the wavelength dependency into account. The group refractive in$\operatorname{dex}\left(n_{g r}\right)$ is calculated as

$$
n_{g r}=n_{e f f}-\lambda \frac{d n_{e f f}}{d \lambda}
$$

Typical mode profiles for the rib and photonic wire wave-
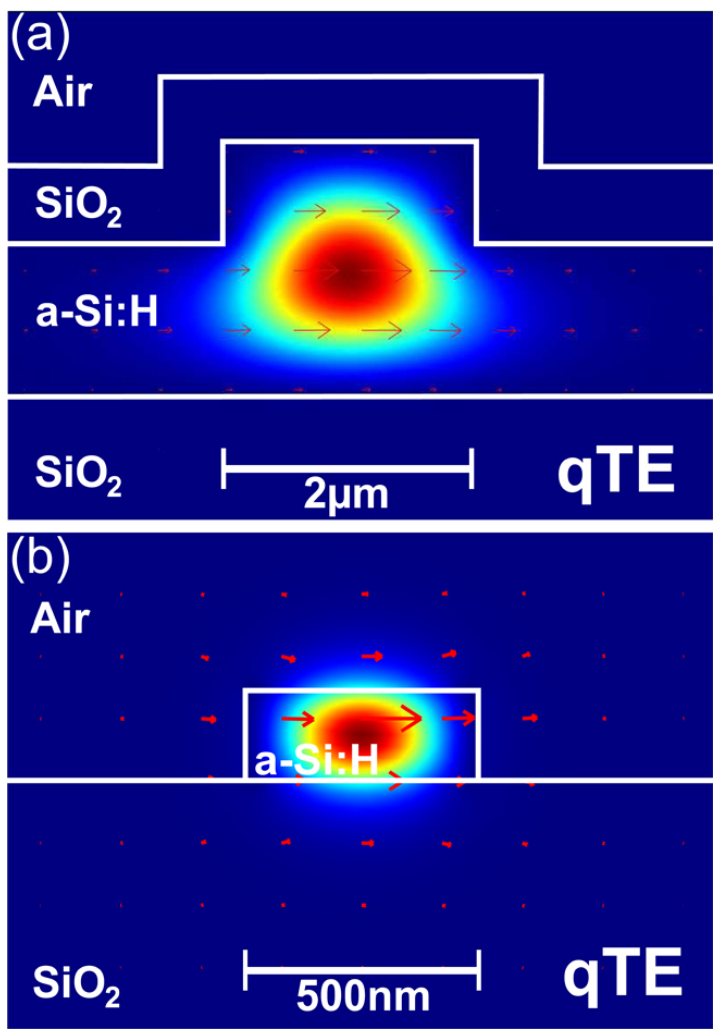

FIG. 1 Fundamental qTE mode profiles of rib (a), and photonic wire waveguides (b). The arrows indicate the electric field.

guides that were obtained for the fundamental qTE mode are presented in Figure 1.

\subsection{Fibre-to-Chip Couplers, Resonators, and Interferometers}

Fibre-to-waveguide couplers, resonators, and interferometers are essential key building blocks of integrated photonic circuits. In this paper, a robust three-dimensional etchless taper is presented, which allows an adiabatic coupling from a riblike facet to the photonic wire and can be matched to the optical fibre mode field. The design process with a line-of-sight model and the fabrication sequence is described more precisely in [16]. Here, the method is extended to polarisation insensitive photonic wires. The real deposition profile of the taper layer was measured with ellipsometry, validated with an alpha-step profilometer, and fed to the FEM-mode solver for a detailed investigation. The simulation results prove that such a taper can be effectively used to end-fire couple light into either polarisation maintaining $(500 \times 220 \mathrm{~nm})$ or polarisation insensitive photonic wires (306x340 nm for an air cladding). The real taper shape is shown for better illustration purposes in Figure 2.

The effective index mode evolutions along the propagation direction of the 3D-taper, which were computed by slicing the taper perpendicularly to that, are summarised in Figure 3. The graph shows that a smooth transition is achieved for both kinds of photonic wire types.

Mach-Zehnder-interferometers (MZIs) are commonly used as broadband spectral filters, for phase measurements e.g. as 


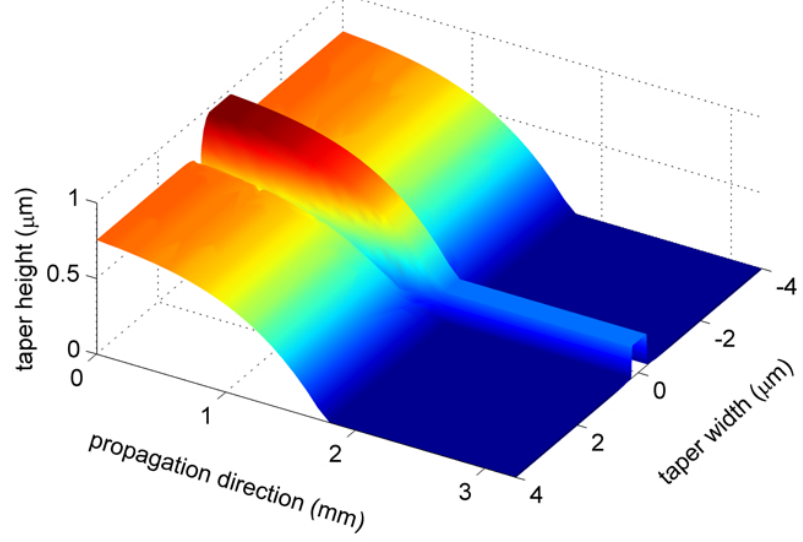

FIG. 2 Three-dimensional shape of the fabricated etchless taper.

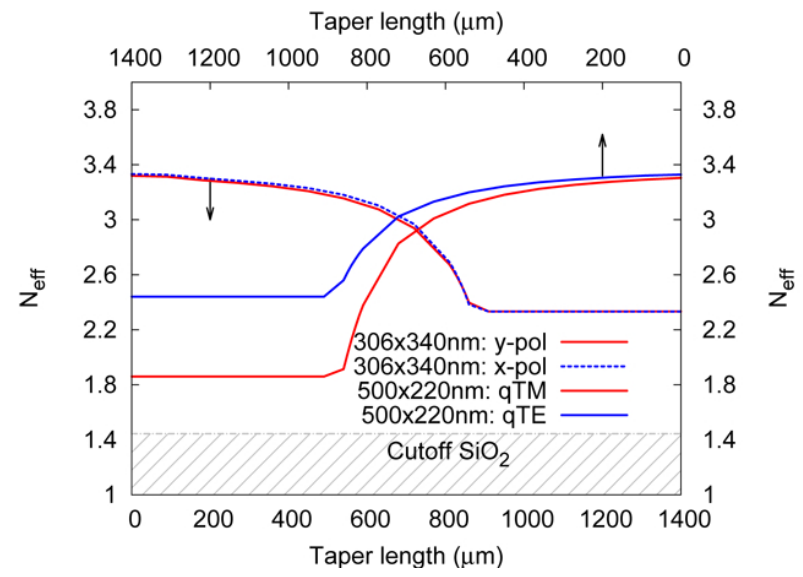

FIG. 3 Effective index mode evolution of two different waveguide dimensions along the tapered photonic wire region for both polarisations.

a sensor, or if electrically connected as modulator by either changing the amplitude or the phase in one of the MZI-arms $[13,17,18]$. In our design multimode interference (MMI) couplers were used. Compared to directional couplers the fabrication tolerances of MMIs are higher and make a precise splitting ratio of both polarisations easier. The MMIs were designed to have a 3-dB splitting ratio for the fundamental qTE- and qTM-modes in C-band and have a low footprint of $\approx 3 \times 8 \mu \mathrm{m}^{2}$. The MMI dimensions were analysed with parametric simulations optimizing width, length, and output arm distance using plane wave propagation as presented in Figure 4 . For a length of $8.25 \mu \mathrm{m}$ and an output waveguide separation of $1.15 \mu \mathrm{m}$ the optimal footprint was determined achieving 50\% splitting. The difference in MZI arm length was chosen to be $\Delta L=200 \mu \mathrm{m}$, which corresponds to a free spectral range (FSR) of 2.76 and 3.44 for qTE and qTM, respectively.

Microring resonators (MRRs) are extremely versatile photonic components, which can be employed in many application fields as spectral filter, modulator, multiplexer and demultiplexer, or as a refractive index sensor $[19,20]$. Due to the high refractive index of a-Si:H a large free spectral range of $18 \mathrm{~nm}$ is achieved for a ring with $5 \mu \mathrm{m}$ radius. In this work, $10 \mu \mathrm{m}$ ring resonators were fabricated as notch filter and in a MZIassisted arrangement, which allows to increase the free spectral range significantly and to reconfigure and tune the res-

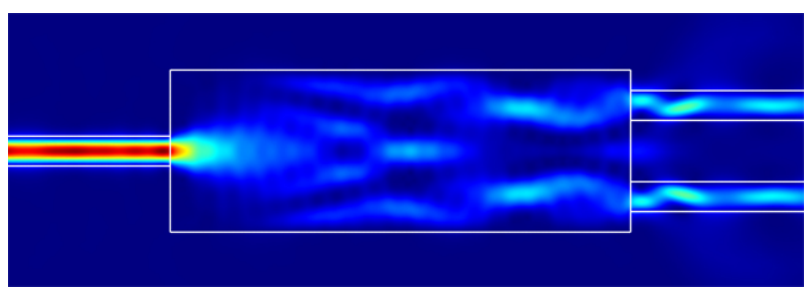

FIG. 4 Simulation of plane wave propagation for the $3 \mathrm{~dB}$ multimode interference couplers showing the averaged powerflow.

onance peak and bandwidth if thermally excited [21, 22]. The design parameters of the photonic devices presented throughout this work and the corresponding key data are summarized in Table 1.

\section{MATERIAL CHARACTERISATION AND FABRICATION}

\subsection{High Index Thin Film Deposition and Characterisation}

For crystalline silicon based SOI wafers the refractive index is fixed at 3.476 (for $1550 \mathrm{~nm}$ wavelength), which is due to the ordered crystalline lattice structure. Compared to that, a$\mathrm{Si}: \mathrm{H}$ can be deposited in various ways by tuning the process conditions. Thereby the refractive index can be varied as a function of hydrogen concentration and $\mu$-crystalline content. Among different a-Si:H deposition methods the lowtemperature PECVD process proved to be most beneficial for the fabrication of low-loss optical material $[4,5,8,23]$.

In this work, the photonic a-Si:H thin films were deposited with a low frequency PECVD system using $380 \mathrm{kHz}$ frequency and $\mathrm{SiH}_{4} / \mathrm{Ar}$ plasma. The parallel plate reactor operates in a low self-bias configuration, grounded bottom and floating top electrode, which effectively reduces high ion bombardment towards the substrate. A low defect density was tailored by using a moderate deposition temperature between 250 and $300^{\circ} \mathrm{C}$, furthermore dangling bonds were sufficiently saturated during the process by choosing an appropriate gas dilution in combination with adapted deposition power and pressure [5].

Due to the released thermal budget $\left(\leq 300^{\circ} \mathrm{C}\right)$, the process temperature is low enough that a variety of different substrate materials like glasses can be used. As a proof of concept, a$\mathrm{Si}: H$ thin films were deposited on different glass substrate materials as shown in Figure 5. However, care has to be taken to keep a low surface roughness, which was recently demonstrated for conductive indium tin oxide (ITO)-layers by using an intermediate spin-on-glass layer as reported in [24].

Ellipsometry was used to determine the thickness and refractive indices of the amorphous silicon layers, which were deposited on grown thermal silicon dioxide. The reproducibility of refractive index and thickness was studied for ten wafers resulting in $n(1534 \mathrm{~nm}) \approx 3.479 \pm 0.24 \%$ and $t \approx 200 \mathrm{~nm} \pm 0.64 \%$, respectively. The typical uniformity for a single wafer was determined by 81 points measurement and 


\begin{tabular}{|l|l|c|c|c|c|c|}
\hline Device & $\begin{array}{l}\mathbf{w} \mathbf{x} \text { h } \\
(\mu \mathrm{m})\end{array}$ & $\begin{array}{c}\boldsymbol{n}_{\text {eff }} \\
\mathrm{TE} / \mathrm{TM}\end{array}$ & $\begin{array}{c}\boldsymbol{n}_{\text {group }} \\
\mathrm{TE} / \mathrm{TM}\end{array}$ & Length & $\begin{array}{c}\text { Cladding } \\
\text { refractive index }\end{array}$ & $\begin{array}{c}\text { FSR (nm) } \\
\mathrm{TE} / \mathrm{TM}\end{array}$ \\
\hline MZI & $0.5 \times 0.22$ & $2.39 / 1.58$ & $4.358 / 3.496$ & $\Delta L=200 \mu \mathrm{m}$ & 1 & $2.76 / 3.44$ \\
Ring & $0.48 \times 0.2$ & $2.37 / 1.74$ & $4.09 / 3.0$ & $R=10 \mu \mathrm{m}$ & 1.6 & $9.34 / 12.71$ \\
MZI-RR & $0.48 \times 0.2$ & $2.37 / 1.74$ & $4.09 / 3.0$ & $\Delta L=60 \mu m$ & 1.6 & $9.79 / 13.35$ \\
& & & & $R=10 \mu m$ & & $9.34 / 12.71$ \\
\hline
\end{tabular}

TABLE 1 Design parameters of the fabricated photonic devices including geometries, FSRs and group refractive indices at $1550 \mathrm{~nm}$ wavelength.

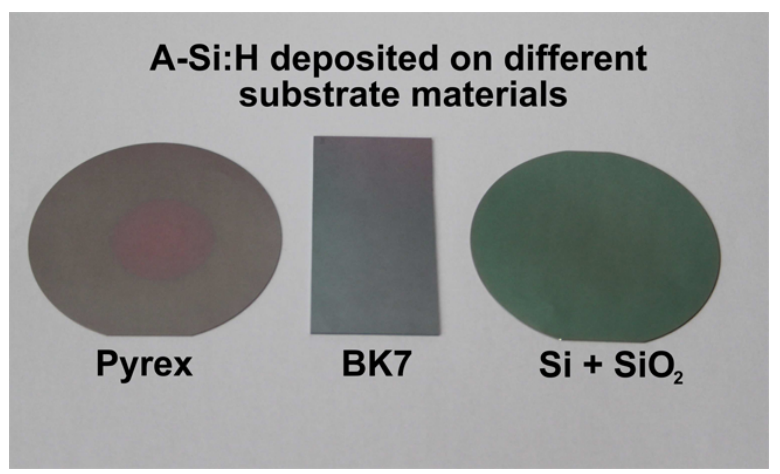

FIG. 5 Photo of a-Si:H deposited on different substrate materials: Pyrex, BK-7 glass, and thermal silicon dioxide grown on standard silicon wafers.

a relative standard deviation of $n<0.25 \%$ and $t \approx 3 \%$ was achieved over the whole wafer when omitting the outermost $1 \mathrm{~cm}$. A systematic investigation is beyond the scope of this paper and will be published elsewhere.

The surface roughness of the thermal oxidised bottom oxide $(t=3 \mu \mathrm{m})$ and the deposited and unpolished a-Si:H core layers $(t=200 \mathrm{~nm})$ on top were measured with atomic force microscope (AFM). A typical AFM surface topography measured in tapping-mode on a $20 \times 20 \mu \mathrm{m}$ scan field yielded smooth layers with $\sigma_{r m s} \approx 0.25 \mathrm{~nm}$ and $\sigma_{r m s} \approx 1 \mathrm{~nm}$ of random mean square roughness, and is shown in Figure 6. The measurement was repeated across and on different a-Si:H wafers which were deposited with the same process conditions using the same layer thickness and the roughness was comparable. SOI, which recieves a polishing treatment, typically has a surface roughness of $\sigma_{r m s} \approx 0.25 \mathrm{~nm}$ on a similar scan area.

\subsection{Photonic System Fabrication}

Standard crystalline silicon wafers $(10 \mathrm{~cm})$ with $3 \mu \mathrm{m}$ wet grown thermal oxide were used as substrates. Thus, substrate leakage is effectively avoided for both fundamental modes.

The PECVD chamber was cleaned with a $C F_{4} / \mathrm{O}_{2}$ based plasma and was pre-conditioned with at least $1 \mu \mathrm{m}$ of the optimised a-Si:H material in order to achieve stable plasma conditions prior the actual core layer deposition. Then the a-Si:H core layers were deposited, $2.1 \mu \mathrm{m}$ for the rib, $200 \mathrm{~nm}$ and $220 \mathrm{~nm}$ for the photonic wire waveguides, respectively.

In case of the rib waveguides i-line lithography (365 nm) was applied. The etching was done with a "Bosch"-process with alternating steps of etching and passivation using 18 cycles targeting a rib etch depth of $900 \mathrm{~nm}$. The photonic wire based

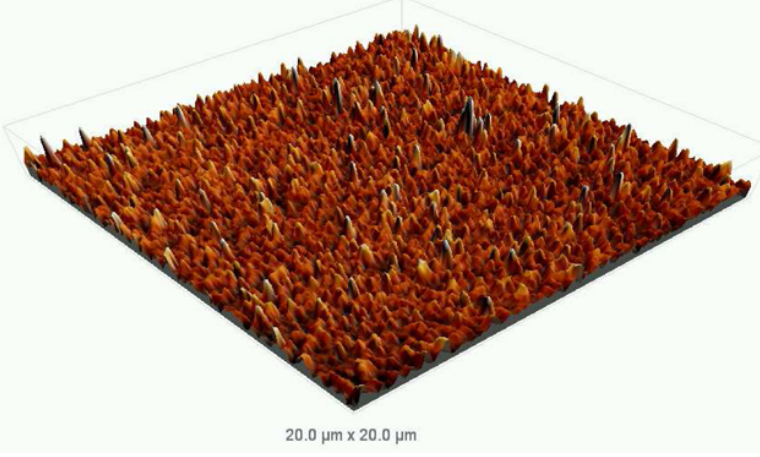

FIC. 6 Surface roughness plot of an a-Si:H photonic thin film measured with atomic force microscope on a $20 \times 20 \mu \mathrm{m}$ scan field.

MZIs were patterned with DUV-lithography at $\lambda=248 \mathrm{~nm}$ and a nm-precise thick bottom anti-reflection coating (BARC).

First the photo resist was developed, the BARC was etched with an $\mathrm{CF}_{4} / \mathrm{O}_{2}$ plasma and the a-Si:H core layer was structured by continuous dry etching with $S F_{6}$ and $C_{4} F_{8}$ in an inductively coupled plasma (ICP). The ring resonators were written with electron-beam lithography at HHI-Berlin in order to achieve precise and small gaps down to $200 \mathrm{~nm}$ in the coupling region of the bus and the ring waveguide. A short descum process with $\mathrm{O}_{2}$-plasma was applied in order to smooth the ZEP-520 photo resist, followed by ICP dry etching.

After photonic system patterning the wafers were exposed to an additional $\mathrm{O}_{2}$-plasma in order to remove the remaining photo resist and BARC layers. Some of the photonic wires were tapered by using a $\mathrm{KOH}$-etched shadow mask, which was manually aligned at the wafer flat and placed face down onto the wafer. The wafer stack was supplied to the PECVD chamber and the deposition of the actual taper was performed. In a last step, the photonic chips were cleaved and cleaned with an ultrasonic bath sequence of isopropanol, acetone, and deionised water rinsing.

\section{EXPERIMENTS AND DISCUSSION}

\subsection{Measurement Setup}

Closed-loop controlled piezo-electric alignment stages were used to adjust the coupling. The NIR-light from the laser (Agilent 8064A) was amplified by an EDFA (C-band) and supplied to a polarisation synthesizer, which set the TE- and TMpolarisations of the probe light. Via lensed fibre, the light was launched into the optical waveguides and microscope objec- 


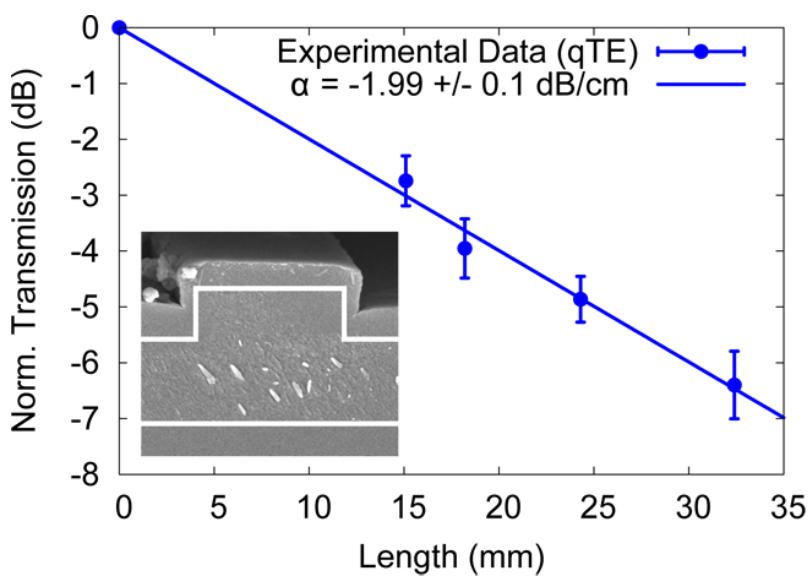

FIG. 7 Cut-back measurement of single-mode a-Si:H rib waveguides with scanning electron microscope picture inset.

tives (up to numerical apertures of 0.85 ) were used to collect the output light and were fed to a fibre-coupled collimator. As detector, an InGaAs photodiode was data-logged with an oscilloscope. A polariser was inserted into the outcoupled beam to ensure the right settings, thereby achieving a polarisation extinction ratio $(E R)$ of $\approx 30 \mathrm{~dB}$ during the experiments. The ring resonator based photonic systems were coupled with qTE polarisation via grating couplers from the top.

\subsection{Waveguide and Taper Characterisation}

The waveguide propagation losses of the rib waveguides were determined with several cut-back measurements with chips from across the whole wafer taking at least 10 measurements for each length. The wavelength was fixed at $1550 \mathrm{~nm}$. The average propagation loss of the waveguides was determined from a linear fit to be $1.99 \pm 0.1 \mathrm{~dB} / \mathrm{cm}$. The deviation per length was about $\pm 0.5 \mathrm{~dB}$ as indicated by the error bar in Figure 7 . The measurements were verified with scattering light measurements by detecting the scattered light of the waveguide from the top. The results were comparable. The best waveguides showed a propagation loss of $\approx 1 \mathrm{~dB} / \mathrm{cm}$. We expect that the differences in propagation loss are mostly attributed to the fabrication tolerances, e.g. the cleaved waveguide facets that were not polished and the sidewall roughness, which resulted from the "Bosch"-etch process, and not to material bulk loss variations.

In case of photonic wires with 500x200 nm dimensions the propagation loss, measured with virtual cutback method, was determined to be $5.26 \pm 0.3 \mathrm{~dB} / \mathrm{cm}$ for qTE mode as presented in Figure 8. The loss is higher than for the rib waveguides and is mainly attributed to roughness at the sidewalls and the optical layer interfaces, which was identified by numerical scattering loss calculations and from locally corresponding scattering peaks of straylight $[11,25]$. The waveguides are folded and consist of $32-90^{\circ}$ bends of $5 \mu \mathrm{m}$ radius and hence allow to extract the bending loss from the interception of the linear fitting with the ordinate. This results in $0.81 \pm 0.06 \mathrm{~dB}$, and when assuming a statistical deviation for each bend, accordingly a loss per bend of $\frac{0.81}{32} \pm \frac{0.06}{\sqrt{32}}=0.025 \pm 0.01 \mathrm{~dB} / 90^{\circ}$ was calculated. The propagation and bend loss measurement results are summarised in Table 2.

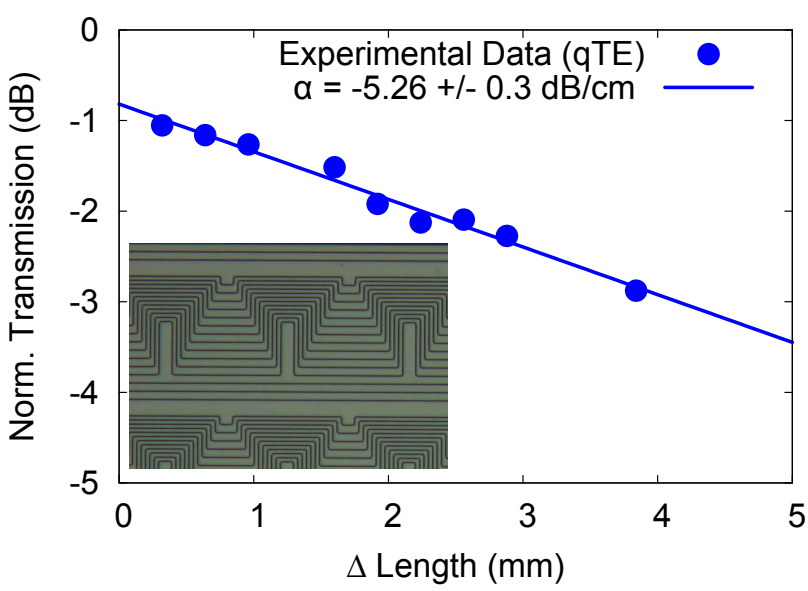

FIG. 8 Virtual cutback measurement of folded photonic wires with different length and 32 bends of $5 \mu \mathrm{m}$ radius. A light microscopic picture is inset.

\begin{tabular}{|c|c|c|c|}
\hline Guide & $\begin{array}{c}\mathbf{w} \mathbf{x} \mathbf{h} \\
(\mu \mathrm{m})\end{array}$ & $\begin{array}{c}\boldsymbol{\alpha}(\mathrm{dB} / \mathrm{cm}) \\
\mathrm{qTE}\end{array}$ & $\begin{array}{c}\text { bend loss } \\
\left(\mathrm{dB} / 90^{\circ}\right)\end{array}$ \\
\hline Rib & $2.1 \times 2.1$ & $1.99 \pm 0.1$ & $\mathrm{x}$ \\
Wire & $0.5 \times 0.2$ & $5.26 \pm 0.3$ & $0.025 \pm 0.01$ \\
\hline
\end{tabular}

TABLE 2 Loss measurement results at $1550 \mathrm{~nm}$ ( $\mathrm{qTE}$ ) of the photonic waveguides and $90^{\circ}$ bend loss for $r=5 \mu \mathrm{m}$ in case of photonic wires.

The 3D-tapers, which were deposited on top of the prior structured photonic wires (500x220 nm), were characterised by insertion loss measurements. First completely untapered photonic wires were measured as a reference. Then the light transmission of tapered photonic wires were determined and the coupling loss was calculated from both measurements. The average coupling loss of five tapers was $4 \pm 0.5 \mathrm{~dB} /$ port including a $1.5 \mathrm{~dB} /$ port field mismatch between lensed fibre and taper. From scattering light measurements the remaining loss source was identified to be at the end of the taper and can be further reduced by an additional lateral taper in the photonic wire plane as reported in [26], or by a slight overetch of the buried oxide cladding.

\subsection{Photonic Device Characterisation}

The power splitting of the 1x2 MMIs were investigated for both polarisations in detail using ten samples. Therefore, light was launched into the MMI input and the power at the two output arms was monitored at the output facet. The results showed splitting ratios of $0.49-0.51 \pm 0.064$ for $\mathrm{qTE}$ and $0.488-0.512 \pm 0.048$ for qTM modes with deviations in mean output power for both ports of $\pm 0.45 \mathrm{~dB}$ and $\pm 1.4 \mathrm{~dB}$, respectively. The excess loss of the MMIs, compared to the maximal achievable power efficieny obtained by the simulations, were determined by insertion loss measurements by quantitative comparison with straight waveguides of the same length and provided $1 \mathrm{~dB}$ in case of qTE and about $2 \mathrm{~dB}$ for qTM.

The MZIs and the MRRs were characterised with spectral measurements by sweeping the tuneable laser wavelength. The results of the MZI are summarised in Figure 9. The difference in MZI arm length was chosen to be $\Delta L=200 \mu \mathrm{m}$. The average FSRs in the spectral measurement range are 2.73 and 3.33 for qTE and qTM-mode, which according to 


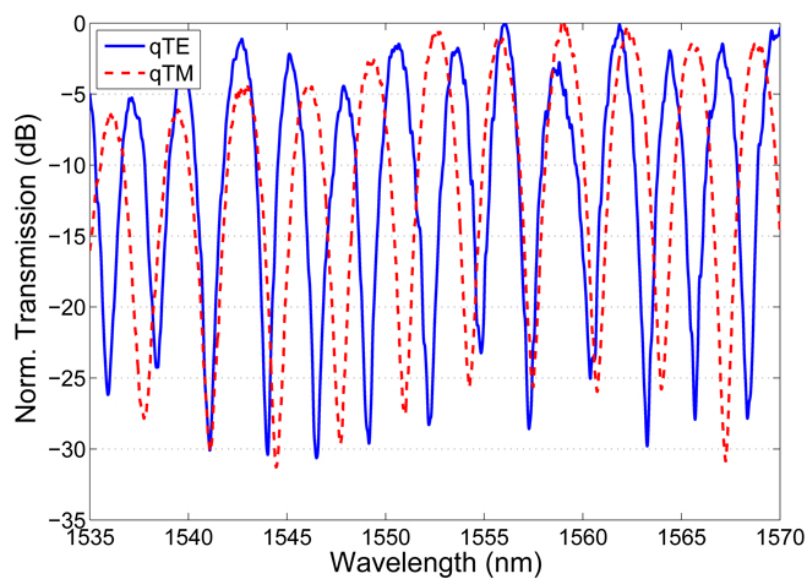

FIG. 9 Transmission spectrum of a MMI-based MZI for qTE and qTM polarisations.

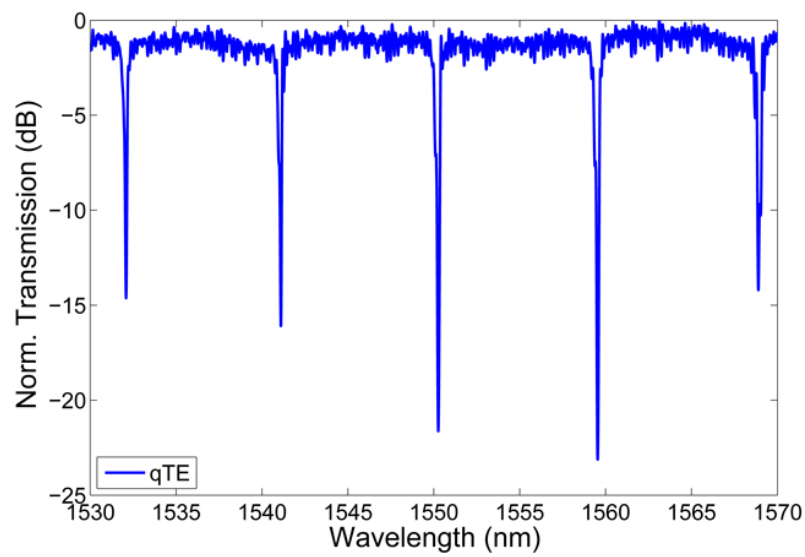

FIG. 10 Transmission spectrum of a $10 \mu \mathrm{m}$ ring resonator with a $200 \mathrm{~nm}$ gap for qTE-mode.

$n_{g r}=\frac{\lambda_{\min } \cdot \lambda_{\max }}{2 \cdot \Delta L \Delta \lambda}$ results in an experimentally determined group index $n_{g r} \approx 4.39$ for qTE and $n_{g r} \approx 3.6$ for qTM. The group indices agree well with the simulations, and the extinction ratios $>25 \mathrm{~dB}$ of the interference fringe pattern confirm the low photonic wire loss and the polarisation insensitive $3 \mathrm{~dB}$ splitting of the MMIs.

The optical measurement of a $10 \mu \mathrm{m}$ photonic ring resonator with a gap of $200 \mathrm{~nm}$ is presented in Figure 10. The MRRs are cladded with a polymer with $n=1.6$ in order to increase the modal overlap of ring and photonic wire. The free spectral range of the resonator is $F S R \approx 9.16 \mathrm{~nm}$ and an extinction ratio $\geq 20 \mathrm{~dB}$ was achieved. The loaded quality (Q)-factor of the ring is calculated according to

$$
Q_{\text {loaded }}=\frac{\lambda_{\text {res }}}{\Delta \lambda_{3 d B}},
$$

where $\lambda_{\text {res }}$ is the resonance wavelength and $\Delta \lambda_{3 d B}$ the $3 \mathrm{~dB}$ bandwidth of the resonance peak. Quality factors of around 7500 were determined with a group refractive index of $n_{g r} \approx 4.17$.

The MZI-coupled ring resonator consists of a $10 \mu \mathrm{m}$ ring with a MZI arm which has a length difference of $\Delta L \approx 60 \mu \mathrm{m}$ with respect to the ring path. The spectral response for the through and the drop port is presented in Figure 11. As expected, only one ring resonance at $1534 \mathrm{~nm}$ is notably excited which is due to the MZI loading effect, whereas the other resonances are

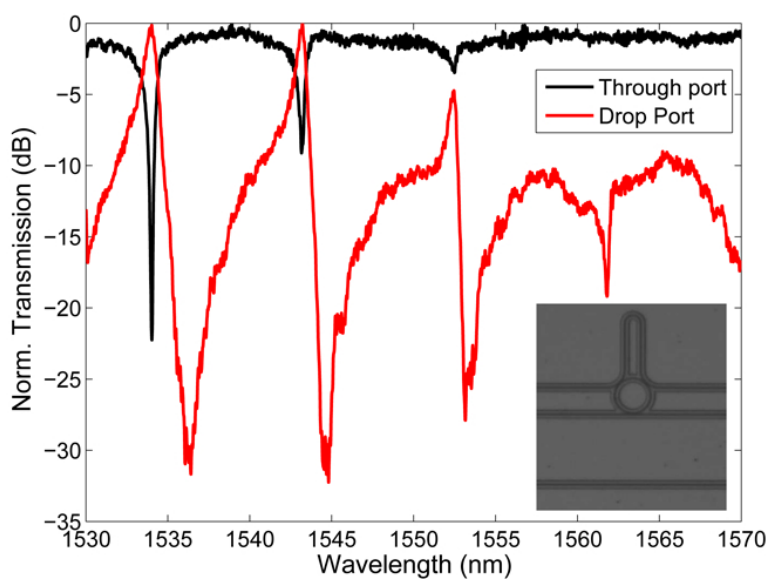

FIG. 11 Through and drop port transmission spectra of a Mach-Zehnder coupled $10 \mu \mathrm{m}$ ring resonator with picture inset.

\begin{tabular}{|c|c|c|c|}
\hline Device & $\begin{array}{c}\mathbf{w} \times \mathbf{h} \\
(\mu \mathrm{m})\end{array}$ & $\begin{array}{c}\text { FSR }(\mathbf{n m}) \\
\text { TE/TM }\end{array}$ & $\begin{array}{c}\boldsymbol{n}_{\text {group }} \\
\mathrm{TE} / \mathrm{TM}\end{array}$ \\
\hline MZI & $0.5 \times 0.22$ & $2.73 / 3.33$ & $4.39 / 3.6$ \\
Ring & $0.48 \times 0.2$ & 9.16 & 4.17 \\
MZI-RR & $0.48 \times 0.2$ & 9.13 & 4.18 \\
\hline
\end{tabular}

TABLE 3 Data of the fabricated photonic devices including geometries, measured FSRS and group refractive indices.

strongly suppressed. The ring resonator FSR is 9.13 which corresponds to $n_{g r} \approx 4.18$. The extinction of the main peak is $20 \mathrm{~dB}$ and the bandwidth of the resonance peak is $\approx 0.5 \mathrm{~nm}$ corresponding to a $\mathrm{Q}$ of 3000 . The ring is slightly overcoupled which can be seen from the asymmetric resonance peak and could be readjusted e.g. by thermal heating of the MZI-arm. The MZI extinction is $30 \mathrm{~dB}$ in case of resonance condition.

The measurement results of this section including the photonic wire dimensions and the experimentally determined FSRs and the group indices for the presented photonic devices are given in Table 3 . The values show a good agreement with the design parameters and underline the reliability of the aSi:H deposition process and the ellipsometric measurements.

\section{$4 \cdot 4$ Discussion}

The photonic devices which are presented in this work show that a-Si:H material provides a flexible and low optical loss platform for high index photonic systems. Basic waveguides as rib and photonic wire waveguides show comparable performance as their crystalline counterparts when using similar process equipment. However, the PECVD fabrication process shows a higher flexibility which is proven by deposition of a-Si:H onto different substrate materials as glasses like BK-7 and Pyrex, and the fabrication of unique type 3D-tapers which serve as robust, efficient and broadband fibre-to-waveguide couplers. The layers show a high uniformity and reproducibility of thickness and refractive index. Furthermore, the process is CMOS-compatible and can be applied on top of current integrated electronic circuits at the last process step after the metallization layers as the deposition temperature is low enough. 


\section{CONCLUSIONS}

In this paper, the a-Si:H material deposition and fabrication technology was optimised in order to achieve high refractive index and low-loss photonic waveguides. The PECVDprocess was analysed in intra-wafer and wafer-to-wafer experiments showing low process deviations and an uniformity of $\leq 1 \%$ for refractive index and $\leq 3 \%$ for the thickness, respectively.

A fabrication concept of three-dimensional tapers for the light coupling into either polarisation independent or maintaining photonic wires is presented, where the coupling loss was measured to be $4 \mathrm{~dB}$ / port for $500 \times 220 \mathrm{~nm}$ photonic wires.

Multimode $3 \mathrm{~dB}$-splitters were systematically investigated resulting in $49-51 \%$ splitting ratios and Mach-Zehnder interferometers based on these splitters showed interference fringe depths of up to $25 \mathrm{~dB}$ for qTE and qTM-polarisations. Compact ring resonators with $10 \mu \mathrm{m}$ radius which were critically coupled and were implemented in a notch filter and in a Mach-Zehnder coupled configuration provided extinction ratios of $\geq 20 \mathrm{~dB}$ and Q-factors up to 7500 .

The results prove that a-Si:H is well suited for multileveled photonic circuits, which could be effectively combined with microelectronics at the backend, and can be used for future on-chip applications in the area of telecommunication, computing, and sensing.

\section{ACKN OWLEDGEMENTS}

The authors would like to thank German Research Foundation (DFG grant FOR-653) for funding. Ralf Steingrüber from Fraunhofer Heinrich Hertz Institute (HHI-Berlin) is acknowledged for performing the electron-beam lithography in case of the ring resonator based photonic systems.

\section{References}

[1] J. Schmidtchen, A. Splett, B. Schuppert, K. Petermann, and G. Burbach, "Low loss singlemode optical waveguides with large cross-section in silicon-on-insulator," Electron. Lett. 27, 1486-1488 (1991).

[2] K. Preston, B. Schmidt, and M. Lipson, "Polysilicon photonic resonators for large-scale 3D integration of optical networks," Opt. Express 15, 17283-17290 (2007).

[3] Q. Fang, J. F. Song, S. H. Tao, M. B. Yu, G. Q. Lo, and D. L. Kwong, "Low loss $(6.45 \mathrm{~dB} / \mathrm{cm})$ sub-micron polycrystalline silicon waveguide integrated with efficient SiON waveguide coupler," Opt. Express $16,6425-6432$ (2008).

[4] G. Cocorullo, F. G. D. Corte, I. Rendina, C. Minarini, A. Rubino, and E. Terzini, "Amorphous silicon waveguides and light modulators for integrated photonics realized by low-temperature plasmaenhanced chemical-vapor deposition," Opt. Lett. 21, 2002-2004 (1996).

[5] A. Harke, M. Krause, and J. Müller, "Low-loss singlemode amorphous silicon waveguides," Electron. Lett. 41, 1377-1379 (2005).

[6] S. Zhu, G. Q. Lo, and D. L. Kwong, "Low-loss amorphous silicon wire waveguide for integrated photonics: effect of fabrication process and the thermal stability," Opt. Express 18, 25283-25291 (2010).

[7] R. Sun, K. McComber, J. Cheng, D. K. Sparacin, M. Beals, J. Michel, and L. C. Kimerling, "Transparent amorphous silicon channel waveguides with silicon nitride intercladding layer," Appl. Phys. Lett. 94, 141108-3 (2009).

[8] S. K. Selvaraja, E. Sleeckx, M. Schaekers, W. Bogaerts, D. V. Thourhout, P. Dumon, and R. Baets, "Low-loss amorphous silicon-on-insulator technology for photonic integrated circuitry," Opt. Commun. 282, 1767-1770 (2009).

[9] K. Narayanan, A. W. Elshaari, and S. F. Preble, "Broadband all-optical modulation in hydrogenated-amorphous silicon waveguides," Opt. Express 18, 9809-9814 (2010).

[10] F. G. Della Corte, S. Rao, G. Coppola, and C. Summonte, "Electrooptical modulation at $1550 \mathrm{~nm}$ in an as-deposited hydrogenated amorphous silicon p-i-n waveguiding device," Opt. Express 19, 2941-2951 (2011).

[11] T. Barwicz and H. A. Haus, "Three-Dimensional Analysis of Scattering Losses Due to Sidewall Roughness in Microphotonic Waveguides," J. Lightwave Technol. 23, 2719-2732 (2005).

[12] S. Selvaraja, W. Bogaerts, P. Dumon, D. Van Thourhout, and R. Baets, "Subnanometer Linewidth Uniformity in Silicon Nanophotonic Waveguide Devices Using CMOS Fabrication Technology," IEEE J. Sel. Top. Quant. 16, 316-324 (2010).

[13] P. Dumon, G. Priem, L. Nunes, W. Bogaerts, D. Van Thourhout, P. Bienstman, T. Liang, et.al, "Linear and Nonlinear Nanophotonic Devices Based on Silicon-on-Insulator Wire Waveguides," Jpn. J. Appl. Phys. 45 (8B), 6589-6602 (2006).

[14] R. Soref, J. Schmidtchen, and K. Petermann, "Large single-mode rib waveguides in CeSi-Si and Si-on- $\mathrm{SiO}_{2}$," IEEE J. Quantum Elect. 27, 1971-1974 (1991).

[15] S. Pogossian, L. Vescan, and A. Vonsovici, "The single-mode condition for semiconductor rib waveguides with large cross section," J. Lightwave Technol. 16, 1851-1853 (1998).

[16] A. Harke, T. Lipka, J. Amthor, 0. Horn, M. Krause, and J. Müller, "Amorphous Silicon 3-D Tapers for Si Photonic Wires Fabricated With Shadow Masks," IEEE Photonic. Tech. L. 20, 1452-1454 (2008).

[17] S. Janz, A. Densmore, D.-X. Xu, P. Waldron, J. Lapointe, J. H. Schmid, T. Mischki, C. Lopinski, A. Delage, R. McKinnon, P. Cheben, and B. Lamontagne, Silicon Photonic Wire Waveguide Sensors (Springer Science + Business Media, Boston, 2009.

[18] G. R. Zhou, M. W. Geis, S. J. Spector, F. Gan, M. E. Grein, R. T. Schulein, J. S. Orcutt, J. U. Yoon, D. M. Lennon, T. M. Lyszczarz, E. P. Ippen, and F. X. Kärtner, "Effect of carrier lifetime on forwardbiased silicon Mach-Zehnder modulators," Opt. Express 16, 5218-5226 (2008).

[19] K. Preston, S. Manipatruni, A. Gondarenko, C. B. Poitras, and M. Lipson, "Deposited silicon high-speed integrated electro-optic modulator," Opt. Express 17, (2009).

[20] W. Bogaerts, P. De Heyn, T. Van Vaerenbergh, K. De Vos, S. Kumar Selvaraja, T. Claes, P. Dumon, P. Bienstman, D. Van Thourhout, and R. Baets, "Silicon microring resonators," Laser Photonics Rev. 6, 47-73 (2012).

[21] L. Chen, N. Sherwood-Droz, and M. Lipson, "Compact bandwidthtunable microring resonators," Opt. Lett. 32, 3361-3363 (2007).

[22] H. Shen, M. H. Khan, L. Fan, L. Zhao, Y. Xuan, J. Ouyang, L. T. Varghese, and M. Qi, "Eight-channel reconfigurable microring filters with tunable frequency, extinction ratio and bandwidth," Opt. Express 18, 18067-18076 (2010).

[23] D. Sparacin, R. Sun, A. Agarwal, M. Beals, J. Michel, L. Kimerling, 
T. Conway, A. Pomerene, D. Carothers, M. Grove, D. Gill, M. Rasras, S. Patel, and A. White, "Low Loss Amorphous Silicon Channel Waveguides for Integrated Photonics," in Proceedings to Group IV Photonics, 2006. 3rd IEEE International Conference on, 255-257 (IEEE, Ottawa, 2006).

[24] S. Rao, F. G. Della Corte, and C. Summonte, "Low-loss amorphous silicon waveguides grown by PECVD on indium tin oxide," J. Europ. Opt. Soc. Rap. Public. 5, 10039S (2010).
[25] T. Lipka, A. Harke, 0. Horn, J. Amthor, and J. Müller, "Amorphous Waveguides for High Index Photonic Circuitry," in Proceedings to Optical Fiber Communication Conference, OMJ2 (OFC/NFOEC, San Diego, 2009).

[26] K. Shiraishi, H. Yoda, A. Ohshima, H. Ikedo, and C. S. Tsai, "A silicon-based spot-size converter between single-mode fibers and Si-wire waveguides using cascaded tapers," Appl. Phys. Lett. 91, 141120-3 (2007). 